\section{Study of possibility physical interactions antimalarial combination drugs}

\author{
Timbul Partogi H.Simorangkir \\ Indonesian Army's Pharmaceutical \\ Institute, Bandung, Indonesia
}

\begin{abstract}
Identification of solid state to investigate the possibility of physical interaction between Antimalarial Artemisinin Combination Treatment base Artesunate (AS) and Amodiaquine (AQ) by hot contact method Kofler, cold contact method (crystallization reaction) and binary phase diagram confirmation had been carried out. The results of hot contact method Kofler shown the formation a new crystalline habit as a long and thin needle on the contact zone (mixing zone) between AS and AQ. A different melting point was seen in its single component. Cold contact methods between two of supersaturated solution of component $\mathrm{AS}$ and $\mathrm{AQ}$ in methanol solvent also indicated the growth of crystal habit as similar as hot contact method Kofler. Confirmation by biner phase diagram shown the specific diagram for cocrystalline phase. Solid state interaction between AS and AQ was analysed by powder X-ray diffraction, FTIR (Fourier Transformed Infra Red) spectrophotometric, microscopic SEM (Scanning Electron Microscopic) and thermal DTA (Differential Thermal Analysis), TG-DSC (Thermal GravimetryDifferential Scanning Calorimetry). Microscopic analysis by SEM showed significantly the change of habit and morphology of crystal to long and thin needle shaped. The difference of powder X-ray diffraction (PXRD) interferences peaks were observed in addition to PXRD interference peaks of each component and its physical mixtures that proved formation of cocrystalline phase. DSC Thermogram indicated a new endothermic peak corresponding to melting point of a new cocrystalline phase at temperature $160,4^{\circ} \mathrm{C}$.
\end{abstract}

\section{Introduction}

Artemisinin Combination-based therapy $(\mathrm{ACT})$ as the current mainstay of malaria treatment recommended by $\mathrm{WHO}$ as an antimalarial drug is Artesunate (AS) and Amodiaquine (AQ). AS and AQ generally used independently as a combination of drugs (co-blistered) that cause non optimalization of the treatment or noncompliance in the use of drugs. ${ }^{1}$ The use of fixed-dose combination form is very rare. ${ }^{2}$ AS and AQ are incompatible if they prepared in a single dosage form. AS degraded due to several things: moisture content, processing temperature and the possible influence of 4-aminokuinolin. ${ }^{3}$

Intermolecular interaction in binary system most likely to occur in the two materials which similar. Similarities are generally based on the molecular formula and the internal structure or level of crystallinity lattice symmetry. Interactions are often found in pharmaceutical technology including an eutectic mixture, solid solutions (mixed crystals) and molecular compounds (cocrystal). ${ }^{4}$

Combination between active ingredient and excipients can lead to make transformation and solid-solid interactions in physics and chemistry. ${ }^{5}$ Interaction between ingredients in the drug dosage forms can cause the formation of new impurities, problems in the preparation and manufacturing processes, changes in the nature of the physicochemical properties of drug substance. ${ }^{6}$ Intermolecular interaction as a consequence of the physics of binary systems that occur in the combination of AS - AQ is not known clearly.

The aim of study is to reveal the kinds of AS-AQ intermolecular interactions with microscopic identification approach using thermal contact and crystallization reaction. Characterization of the result of interaction was studied by thermal analysis DSC/DTA, FTIR spectroscopic, PXRD crystalography and SEM.

This research started with researching and characterizing each single compound Artesunate and Amodiaquine $\mathrm{HCl}$ finding the possibility of polymorphisms that will provide physical consequences of the ASAQ binary system. Some methods such as evaporation of solvent (methanol, ethanol, chloroform, $\mathrm{n}$ - hexane), cold crystallization method (Freeze Drying in the water solvent) and grinding had been done to produce crystalline forms.

The changing nature of the crystals was observed by means of powder X - ray diffraction (PXRD), thermal analysis DTA/DSC, HSM (Hot Stage Microscope), SEM and FTIR. The next stage was to construct the phase diagram of physical mixture of AS - AQ in various composition. Further characterization of physical mixture by means of PXRD, thermal analysis by DTA/DSC, HSM, SEM and FTIR to analyze the possibility of intermolecular interactions between AS-AQ. With the effort of uncovering, it is expected to be a
Correspondence: Timbul Partogi Haposan Simorangkir, Jl. Gudang Utara 25-26 Bandung 40113 Indonesia.

Tel.: 08122347496

E-mail: tphsimorangkirsimon@gmail.com

Key words: Antimalarial combination treatment, artesunate, amodiaquine $\mathrm{HCl}$, physical interaction.

Conflict of interest: The author declare no potential conflict of interest.

Funding: The work was supported by Indonesian Ministry of Defence.

Acknowledgements: Special thanks to Prof Dr. rer.nat. Sundani N. Soewandhi and Prof. Dr. H. Uekusa, for their assistance and support on this study also the organizer of international conference Infectious Diseases, Biothreats, and Military Medicine (INSBIOMM) for the chance to present my articles.

Conference Presentation: This paper has been presented at international conference Infectious Diseases, Biothreats, and Military Medicine (INSBIOMM) Surabaya 2019 August.27-28

Received for publication: 17 February 2020. Accepted for publication: 1 July 2020.

This work is licensed under a Creative Commons Attribution-NonCommercial 4.0 International License (CC BY-NC 4.0).

(C) Copyright: the Author(s), 2020

Licensee PAGEPress, Italy

Infectious Disease Reports 2020; 12(s1):8761 doi:10.4081/idr.2020.8761

scientific foundation in problem-solving design and manufacturing processes of dosage formulations fixed combination artesunate-amodiaquine $\mathrm{HCl}$.

\section{Material and Methods}

\section{Materials}

Preparation of the sample, FT IR analysis, HSM analysis and TG-DSC analysis were conducted in Lembaga Farmasi Puskesad and Bandung Institute of Technology . DTA analysis, PXRD analysis were conducted in Airlangga University Surabaya, Surabaya Institute of Technology and Tokyo Institute of Technology Japan. SEM analysis was done in Central Research of Geology Bandung. Artesunate was purchased from Haryana, India with bacth no. AS/M-001/07-08, while Amodiaquine hydrochlortide USP was purchased from Mangalam Drugs \& 
Organics Ltd, Mumbai, India with batch no. AMH-113003. All of solvent were purchased from Merck Chemical Indonesia without any purification.

\section{Observation of habit and a mixture of two components}

Saturated solution of each of artesunate (AS) and amodiaquine hydrochloride (AQ) was made in various solvents (methanol, ethanol, n-hexane, chloroform, distilled water), then each glass dripped on different objects and allowed to crystallize again at room temperature along with the evaporation of the solvent. Saturated solution of physical mixture ekimolar AS and AQ dripped on glass objects, allowed to crystallize. The crystallization process and the crystal habit observed by polarizing microscope and digital camera.

\section{Preparation of the physical mixture of Artesunate and Amodiaquine $\mathrm{HCl}$}

Raw materials of artesunate and amodiaquine hydrochloride weighed and mixed in a molar ratio= $(0: 10),(1: 9),(2: 8)$, $(7: 3),(5: 5),(3: 7),(2: 8),(1: 9),(10: 0)$. Physical mixture homogenized by stirring the two materials in a mortar with zalf cart.

\section{Hot Stages method}

Contact method performed under a polarizing microscope equipped with a desk heater electrik (Hot Stage). A specific number of AQ was put on the object glass and covered, then heated to melt, and allowed to crystallize again. AS powder was put on the side of the border the cover glass. The system is heated until the entire AS will be melt, move and contact with the surface of the AQ crystal. Contact area (contact zone) that occurs between solids and fused AQ-AS was observed for growing of a new crystal under a polarizing microscope at 200x magnification and recorded with a digital camera. ${ }^{4}$

\section{Cold Contact method}

Saturated solution of AQ in the solvent dripped on glass objects, waiting to crystallize. After that the same amount of AS saturated solution dripped on the edge of AQ recrystallization region. Crystallization behavior observed at the contact area between the crystal AQ with a solution of saturated AS. Physical interaction was observed with the polarizing microscope at 200x magnification and recorded with a digital camera. $^{7}$

\section{DTA Thermal analysis}

Each materials and binary mixture of various molar ratio of drugs were prepared for DTA analyzing. Heating was performed in the range $30-300^{\circ} \mathrm{C}$ at scan rate $10^{\circ} \mathrm{C} / \mathrm{min}$. Thermogram of DTA results were used to construct of phase diagram of AS-AQ binary mixture.

\section{Powder X-Ray Diffractometry (PXRD) analysis}

The X-ray diffraction patterns were obtained with X-ray diffraction analysis of the powder sample at room temperature by using a Rigaku diffractometer type Rint2500. Measurement conditions as follows: the target metals $\mathrm{Cu}, \mathrm{K} \alpha$ filter, voltage 40 $\mathrm{kV}$, current $40 \mathrm{~mA}$, the analysis performed at 2 theta range of $5-35^{\circ}$. The sample is placed on the sample holder and leveled to prevent particle orientation during sample preparation.

\section{Scanning Electron Microscopy (SEM) analysis}

Powder sample was placed on the sample holder and coated with gold aluminum with a thickness of $10 \mathrm{~nm}$. Samples were then observed various magnification SEM instrument (JEOL, Japan). Voltage was set at $20 \mathrm{kV}$ and $12 \mathrm{~mA}$ current.

\section{Fourier Transform Infrared (FTIR) Spectroscopy}

About $1 \%$ dispersion powder samples in potassium bromide $(\mathrm{KBr})$ was made by mixing the mass with $\mathrm{KBr}$. Infrared spectrum obtained with an infrared spectrophotometer in the range of wave numbers 400 - $4000 \mathrm{~cm}-1$.

\section{Results and Discussion}

Two methods of heat Kofler contact method and the method of crystallization reactions were used for early identification of physical interaction between two components. ${ }^{4,7,8}$ Thermal contact method was first introduced by Lehman and Kofler. ${ }^{9}$ This method is a simple technique to identify the phase behavior in a binary system. In this method, one component (which has a higher melting point that $\mathrm{AQ}$ ) was melted and allowed to solidify again (recrystallization), the second component AS (lower melting point) was placed on the other side of the glass object, heated using a heater (hot stage) which associated with a polarization microscope. At the time of the second component (AS) melting, molten phase component of the AS will diffuse into the solid component of AQ and AQ dissolve most solids in the contact zone between the binary system AQ and AS. Samples were allowed to solidify (recrystallization) at room temperature. After the second component (AS and AQ) solidified, the contact zone was observed back in the polarizing microscope. ${ }^{4}$

Figure 1 shows side $\mathrm{A}$ is the result of the $\mathrm{AS}$ and the melt recrystallization $\mathrm{C}$ is melt

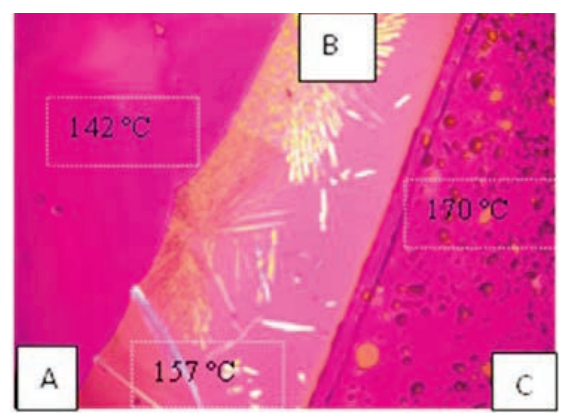

Figure 1. HSM photomicrograph (A) AS area Melting Point (MP) $142^{\circ} \mathrm{C}$; (B) contact zona MP $157^{\circ} \mathrm{C}$; (C) AQ area MP $170^{\circ} \mathrm{C}$.
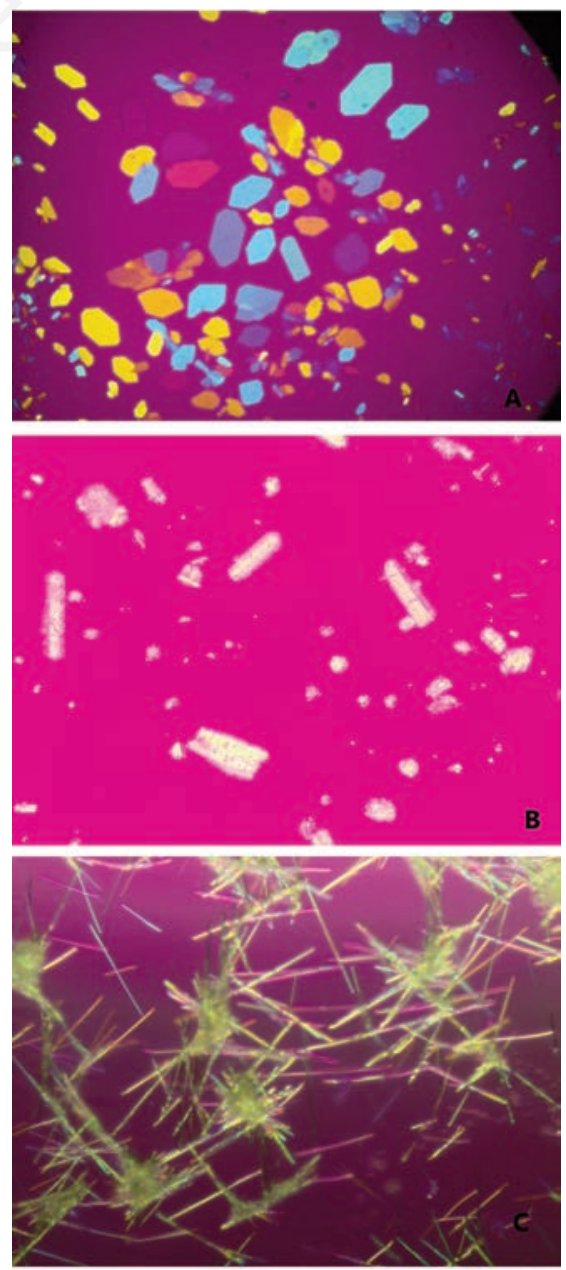

Figure 2. Photomicroscopes of cold contact method, (A) AS in methanol; (B) AQ in methanol; (C) AS-AQ binary mixture in methanol. 
recrystallization $\mathrm{AQ}$. Zone $\mathrm{B}$ is the contact zone between the AS and AQ solids. New crystal habits have not been observed at the beginning of the formation of the contact zone, however, they were still in the liquid phase (amorphous). After settling in a certain time, began to form a new crystal growth habit in zone B-shaped needle (needle shaped habit). Contact method of sample preparation was heated again, then AS solid phase melt at $142^{\circ} \mathrm{C}$, contact zone melt at $157^{\circ} \mathrm{C}$ and $\mathrm{AQ}$ solid phase melt at $170^{\circ} \mathrm{C}$. The difference in crystal habit and thermal behavior of solids indicates the interaction between the two components of the AS and AQ. ${ }^{4,8}$ Three types of solids interaction from the thermal behavior of the molten phase mixture of two components are: (i) conglomerates (eutectic) where both components still exist on a separate crystalline zone, (ii) solid solution in which the two components mixed in a homogeneous solid phase, (iii) cocrystal or molecular compounds which are formed in the solid phase mixing zone that has different properties of the two components. $^{4}$

Crystallization reaction method is also simple method for observing the identification between the two components, especially the drug compounds which unstable on heating. ${ }^{7}$ Each component have good solubility in the methanol solvent. Each component is dissolved in the methanol solvent, until it reaches a state of supersaturation.

AS components and a single AQ dripped on glass object and allowed experienced recrystallization and crystal habit observed in a single state with a polarizing microscope equipped with a digital camera. Super-saturated solution of both components was dropped on the two sides of the glass object to observe the interaction between the AS and AQ binary systems, then the two solutions become contact each another, and then were left for some time at room temperature.

New crystal growth was observed under a polarizing microscope. Figure 2 shows the crystal habit of AS and AQ obtained from recrystallization from methanol solvent and from co-crystallization of both components AS-AQ from the same solvent. Some of the factors that determine the transformation of the solid phase is the nucleation rate, crystal growth rate and distribution of nucleation sites (nucleation sites). Nucleation rate and the growth rate will depend on the state of the supersaturated driving force for the cocrystallization of two solid components from both component molten phase and dissolved phase. ${ }^{7,10}$ To evaluate the results of the contact methods (hot and cold contac), analysis by DTA and the transition temperature data plotted in the phase diagram. AS-AQ phase diagram was made by plotting the melting point (endothermic peak) obtained from thermal analysis DTA with the molar fraction of binary mixture AS-AQ on composition $0: 1 ; 1: 9 ; 2: 8 ; 3: 7$; $5: 5 ; 7: 3 ; 8: 2 ; 9: 1$ and $1: 0$.

Figure 3 is a typical phase diagram between two solid components that interact through the formation of molecular compounds (cocrystal) obtained at a mole fraction of 5:5. Endothermic peak was obtained at a temperature of $158.9^{\circ} \mathrm{C}$, interpreted as a cocrystal melting point. There were two points eutecticum E1 and $\mathrm{E} 2$ at a temperature of $150^{\circ} \mathrm{C}$ and $132.7^{\circ} \mathrm{C}$. AQ melting point decreased with the addition of AS and reached a minimum value at the first eutecticum point (E1) $150^{\circ}$ $\mathrm{C}$ (mole fraction 2:8). From the point of liquidus curve E1 rised due to the addition of more AS and led to increase melting point of the mixture and reached its maximum value at the point of TC. At this point they were in balance with the solid phase liquid phase, where the maximum temperature is congruent compound/ASAQ cocrystal $\left(158.9^{\circ} \mathrm{C}\right)$ on the mole fraction 5:5. Furthermore, the addition of AS will cause a decrease in binary mixture melting point to the minimum point E2 $\left(132.7^{\circ} \mathrm{C}\right)$ at a mole fraction of 9:1. According to figures we can conclude that there was equilibrium between the solid phase with the liquid phase at the same mole fraction composition. This suggests the formation of molecular compounds ASAQ (cocrystal phase) as a consequence of the two components of the binary solids system.

Figure 4 shows the electron microscopic analysis of AS raw materials, AQ and the solid results of the AS-AQ interaction method of cocrystalization reaction of methanol. The solid reaction products (ASAQ cocrystal) showed the different crystal habit and particle sizes compared to each components. The solid interaction (AS-AQ cocrystal) results indicated needle-shaped crystal habit.

The interaction between two solid components of AS - AQ was verified by analysis of X-ray powder diffraction and differential thermal analysis scanning calorimetry (DSC). X-ray diffractogram and DSC thermogram of cocrystal AS-AQ compared with each single component and the physical mixture of both components without treatment (Figures 5 and 6). X-ray powder diffraction is a powerful method for the characterization of the interaction between the two components. When the new crystalline phase was formed from the interaction between the two components, the X-ray diffractogram could be observed clearly.

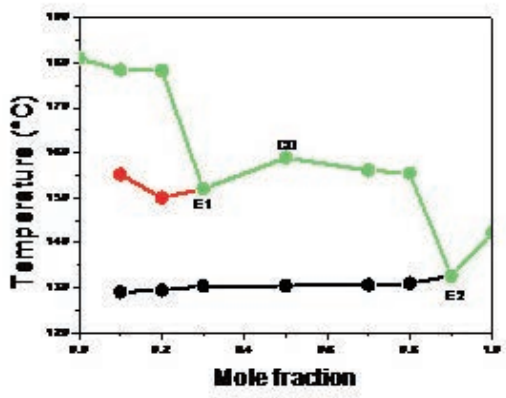

Figure 3. AS-AQ phase diagram. $\mathrm{TA}=$ Melting temperature of $\mathrm{AQ} T \mathrm{~TB}=$ Melting temperature of AS TC=Melting temperature of Cocrystal, E1,E2=Eutectic point,SA=Solid of component $A Q$, SB=Solid of component AS, SC=Solid of component Cocrystal, $\mathrm{L}=$ Liquid.
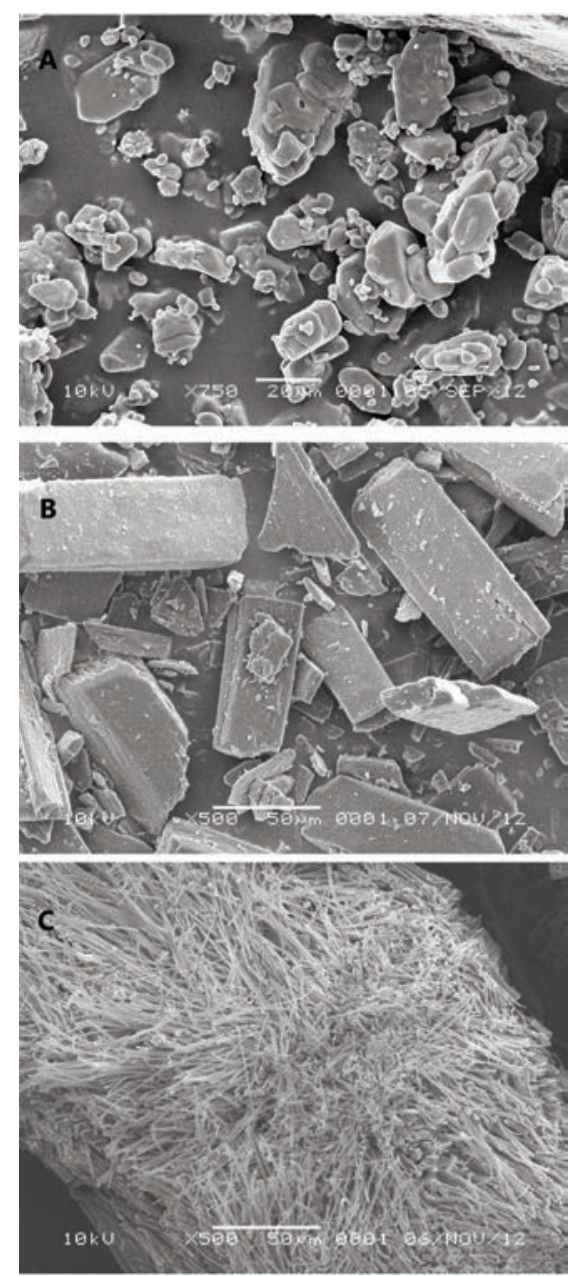

Figure 4. SEM Microphotograph of (A) AS raw materials (750x); (B) AQ raw materials (500x); (C) AS-AQ Cocrystal in methanol solvent $(500 x)$. 
Figure 5 shows the X-ray powder diffractogram of AS-AQ cocrystal compared to the single component and physical mixture of both components without treatment. PXRD pattern of AS AQ cocrystal (Figure 5 D) significantly different from the pattern of diffraction AS,

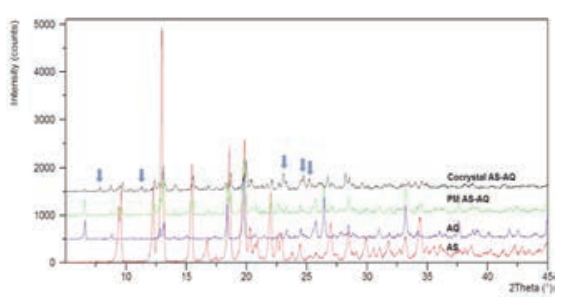

Figure 5. PXRD difractrogram of (A) AS (B) AQ (C) physical mixture AS-AQ (1:1 ekimolar); D) AS-AQ Cocrystal: the new main peak of co crystal.
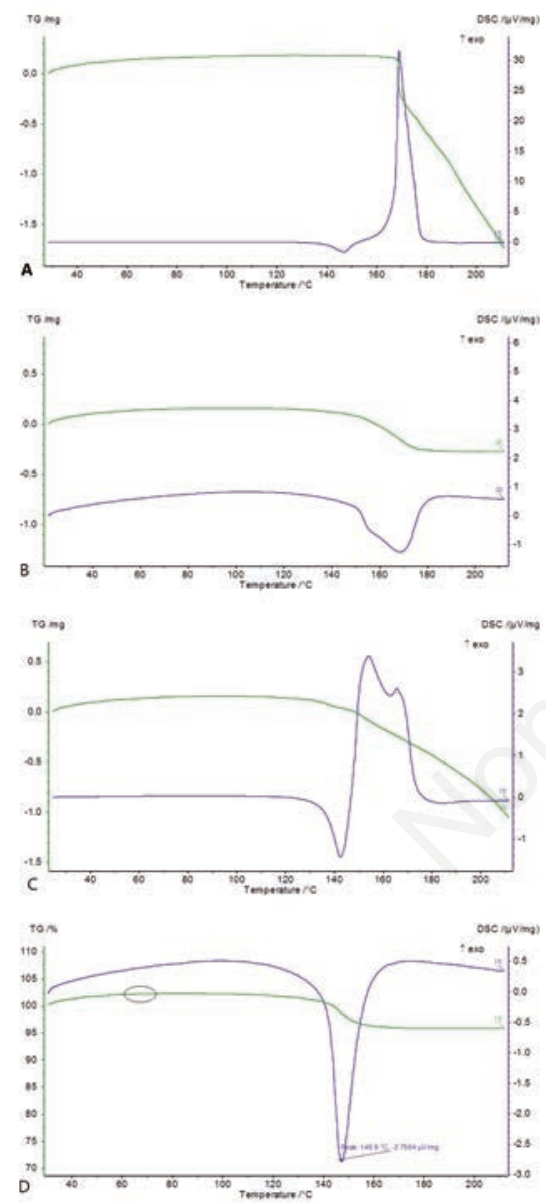

Figure 6. DSC thermogram of (A) AS; (B) AQ; (C) physical mixture AS-AQ (ekimolar); (D) AS-AQ cocrystal.
$\mathrm{AQ}$ and physical mixture of AS-AQ. The diffraction pattern of each component crystalline solids exhibited properties of a single compound. While the diffraction pattern of physical mixture of AS-AQ showed all the typical peak of AS interference and AQ, indicating only superimposition of the two components was occurred. Cocrystal diffraction pattern of AS-AQ showed some new interference peaks typical at $2: 8.77 ; 12.77 ; 23.11 ; 25.28$ and 25.57 , indicated the physical interaction between AS - AQ had formed. The formation result of a new crystalline phase is commonly called cocrystalin phase (molecular compounds or molecular complexes) in materials science

DSC thermal analysis useful for characterizing interactions among multiple components of the solid ingredients. Thermal analysis was used to evaluate changes in thermodynamic properties that occured when the material supplied heat energy. The changes that can be observed during the process of melting were desolvasi, recrystallization and solid phase transformations indicated by endothermic or exothermic peaks at thermogram. DSC thermogram showed AS solids endothermic peak at $142.2^{\circ} \mathrm{C}$ (Figure 6A), while the AQ had a pad endothermic peak $166.8^{\circ} \mathrm{C}$.(Figure $6 \mathrm{~B}$ ). The interaction of MP solids formed from the AS-AQ (cocrystal AS-AQ) related in three endothermic peak at $130.5^{\circ} \mathrm{C} ; 150.5^{\circ} \mathrm{C}$ (eutectic mixture MP) and $158.9^{\circ} \mathrm{C}$, as shown by DSC thermogram of physical mixture ekimolar (Figure 6C). DSC thermogram data for ASAQ crystal showed an endothermic peak at $160.4^{\circ} \mathrm{C}$, indicated that both solid components were transformed into a new crystalline phase of AS-AQ known as cocrystal phase (molecular compounds).

PXRD analysis results showed the formation of a new interference with different physical mixture and some shifts in the FTIR spectrum strengthens the case of AS-AQ cocrystal forming.

\section{Conclusions}

Formation of the solid phase cocrystalin between artesunate and amodiaquine (AS-AQ cocrystal) has been successfully verified by using microscopic methods (hot and cold stages contact methods and SEM micrograph), crystallographic methods (PXRD), thermal methods (DTA / DSC), and spectroscopic methods (FTIR).

\section{References}

1. Beer N, Ali AS, Rotllant G, et al. Adherence to artesunate-amodiaquine combination therapy for uncomplicated malaria inchildren in Zanzibar, Tanzania. Trop Med Int Health. 2009; 14:766-74.

2. Ndiaye JL, Randrianarivelojosia M, Sagara I, et al. Randomized, multicentre assessment of the efficacy and safety of a fixed-dose artesunate-amodiaquine combination therapy in the treatment of uncomplicated Plasmodium falciparum malaria. Malar J 2009; 8:125.

3. Haynes RK. From artemisinin to new artemisinin antimalarials: biosynthesis, extraction, old and new derivatives, stereochemistry and medicinal chemistry requirements. Curr Top Med Chem 2006;6:509-537.

4. Davies RE, Lorimer KA, Wilkowski MA, Rivers JH. Studies of Relationship in Cocrystal Systems. ACA Transactions 2004;39:41-61.

5. Adeyeye MC, Brittain HG. DrugExcipient Interaction Occurences During Solid Dosage Form Development. Informa Healthcare 2008; 178:361-430.

6. Dooren AAV. Design for DrugExcipient Interaction Studies. Drug Development and Industrial Pharmacy 1983;9:43-55.

7. Hornedo NR, Nehm SJ, Seefeldt KF, et al. Reaction Crystallization of Pharmaceutical Molecular Complexes. Mol Pharm 2006;3:362-367.

8. Soewandhi SN.Antar A Qsi Fisik Padatan Pada Kombinasi Senyawa Aprobarbital dan Isopropilantipirina. J M S 1999;4:20-31.

9. Berry DJ, Seaton CC, Clegg W. et al. Applying Hot-Stage Microscopy to Cocrystal Screening: A Study of Nicotinamide with Seven Active Pharmaceutical Ingredients. Crystal Growth and Design 2008;8:1697-1712.

10. Jayasankar A, Somwangthanaroj A, Shao ZJ, Rodriguez-Hornedo N. Cocrystal Formation during Cogrinding and Storage is mediated by Amorphous phase. Pharm Res 2006; 23: 2381-2392. 